\title{
Aggressive Angiomyxoma
}

\author{
Col S Rudra*, Lt Col RN Banerji ${ }^{+}$, Col NS Mani"
}

MJAFI 2007; 63 : 386-387

Key Words: Aggressive angiomyxoma; Pelvic soft tissue tumour

\section{Introduction}

A bdominopelvic soft tissue tumours are common but pelvic retroperitoneal soft tissue tumours are rare. Amongst various soft tissue tumours of abdominopelvis and pelviperineum, aggressive angiomyxoma is a very rare variety of benign neoplasm. This was first described in 1983 by Steeper et al [1]. This mesenchymal tumour arises from connective tissue of lower pelvis or perineum and has a locally aggressive course [2]. About 130 cases of this rare soft tissue tumour have been reported [3].

\section{Case Report}

A 35 year old parous lady was admitted with the complaints of lower abdomen pain and gradually increasing swelling of three months duration. Her menstrual cycles were regular. She was initially managed in civil where she underwent laparotomy. Tumour was found behind the uterus covering the main vessels. Removal was not attempted and the abdomen was closed.

On admission to this hospital clinical examination of the patient revealed a soft mass arising from the pelvis which was about 20 weeks in size, non-tender, with restricted side to side mobility. The uterus was not felt separately from the mass on pelvic examination. Ultrasonography of the abdomen revealed a large heteroechoic fairly well defined mass in the abdominal cavity extending inferiorly into the Pouch of Douglas (POD). CT abdomen showed a hypodense abdominopelvic mass $21 \times 18 \times 8.5 \mathrm{~cm}$, extending superiorly above the bifurcation of the aorta and inferiorly behind the urinary bladder, extending into the POD (Fig.1). The uterus was separate and ovaries were not well defined. Intravenous urography revealed a lateral deviation of left ureter and normal kidney function.

A pre operative clinical diagnosis of soft tissue tumour of pelvis was entertained and laparotomy was performed. The abdomen was opened by a midline suprapubic vertical incision extending up to $5 \mathrm{~cm}$ above the umbilicus. An irregular soft mass $20 \times 20 \times 8 \mathrm{~cm}$ with smooth surface was found arising from behind the uterus in the retroperitoneal space, reaching beyond the aortic bifurcation superiorly and laterally occupying both sides of broad ligament completely. The tubes and ovaries were normal and stretched over the mass. The lower pole of the mass was not visible or palpable as it was occupying the whole of the true pelvis. There were no omental metastases. The surface of the liver was smooth and no para-aortic lymph nodes were palpated. There was no ascitis. Peritoneal washings were taken for cytology.

The approach for excision was made from lateral aspects of the tumour by incising and ligating the infundibulopelvic and round ligaments. The major vessels and ureter were separated from the mass and the anterior horn was dissected out from the base of the bladder wall. The lower pole of the mass was found extending into the rectovaginal space. The mass was mobilized from the bowel wall and simultaneously steps of hysterectomy were continued along with mobilization of the mass. The entire mass along with the uterus, cervix and appendages on both sides were removed along with an upper three centimeter of posterior vaginal wall which was adherent to the lower pole of the mass. Since we did not have facilities for frozen section, omentectomy was also performed and abdomen closed in layers. A postoperative diagnosis of retroperitoneal pelvic soft tissue tumour was made and the specimen was sent for histopathological examination.

On gross examination the tumour was wellcircumscribed with a reddish blue hue externally and weighing $1.2 \mathrm{~kg}$. The cut section showed a solid tumour predominantly yellowish green in colour with central yellow and peripheral gelatinous areas. Histopathological examination showed a predominantly myxomatous tumour with sparsely cellular myxomatous areas and cellular areas adjoining proliferated small and medium caliber muscular blood vessels (Fig.2). Some blood vessels were thin walled and of varying caliber. The neoplastic cells were stellate and spindled. No nuclear pleomorphism was noted. The nuclear chromatin pattern was fine. There was no increased mitotic activity or necrosis seen. Splaying of smooth muscle fibers extending from a few blood vessels was seen. No lipoblasts or nerve sheath elements were seen. Immunohistochemistry showed positivity for Vimentin in the stellate cells and smooth muscle actin (SMA) positivity in spindle cells around muscular blood vessels. The tumour cells were CD 34 positive. No invasion of uterine corpus, 


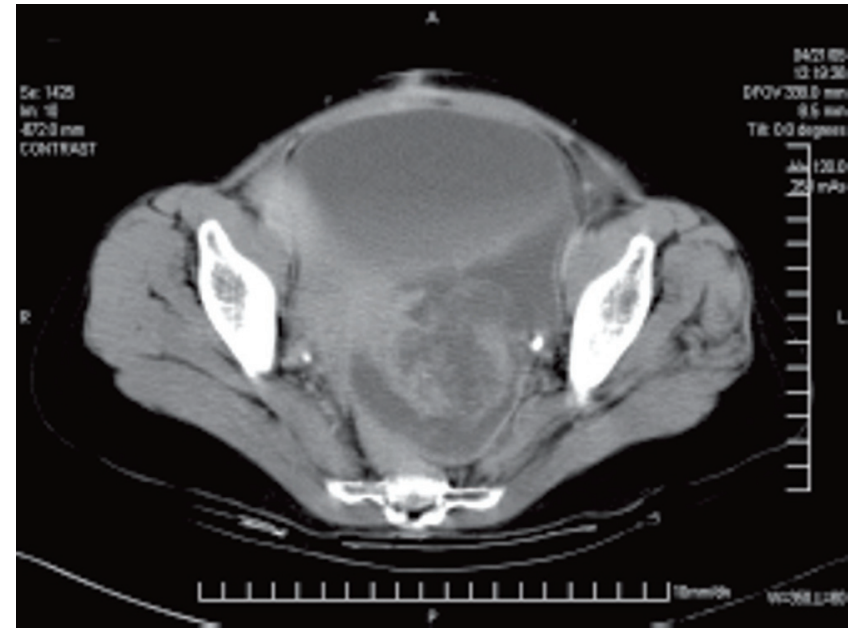

Fig. 1: CT picture showing extent of the tumour almost completely occupying the pelvic cavity

cervix or ovaries was noted. Based on the above findings a diagnosis of aggressive angiomyxoma was made. A one year followup of the case showed no clinical, sonological or CT evidence of recurrence.

\section{Discussion}

Aggressive angiomyxoma is a rare variety of non metastasing soft tissue tumour of pelvis and perineum occurring almost exclusively in adult females (female to male ratio is $6.6: 1$ ). The age distribution is wide, with a peak incidence at 31-35 years. These large sized tumours are slow growing and painless. The presenting features are nonspecific such as a palpable mass with heaviness or discomfort in the lower abdomen. The commonest affected sites involved are pelvis, perineum and vulva.

Complete surgical excision is the gold standard, because of its tendency to recur locally. The recurrence rate is varies from $36-70 \%$. There is no correlation between size of tumour and recurrence rate[4]. Surgery causes significant morbidity due to its frequent occurrence in lower pelvis and perineum with proximity to genitourinary and anorectal structures [2]. Most surgeons aim at complete resection (wide excision with tumour free margin), incomplete or partial resection is acceptable when high operative morbidity is anticipated and fertility is an issue [4]. Treatment with GnRH agonists in oestrogen and progesterone receptors positive tumours obviates repeat surgery for recurrence [5]. Radiation therapy also reduces such recurrences.

Various soft tissue tumours in this region considered for differential diagnosis are angiomyofibroblastoma, angiofibroma, spindle cell lipoma and aggressive angiomyxoma. Angiomyofibroblastoma occurs later in life (3rd to 9th decades) and involves vulva and vagina. It presents as a submucous mass and shows high cellularity, large number of blood vessels and plump stromal cells with perivascular accentuation on

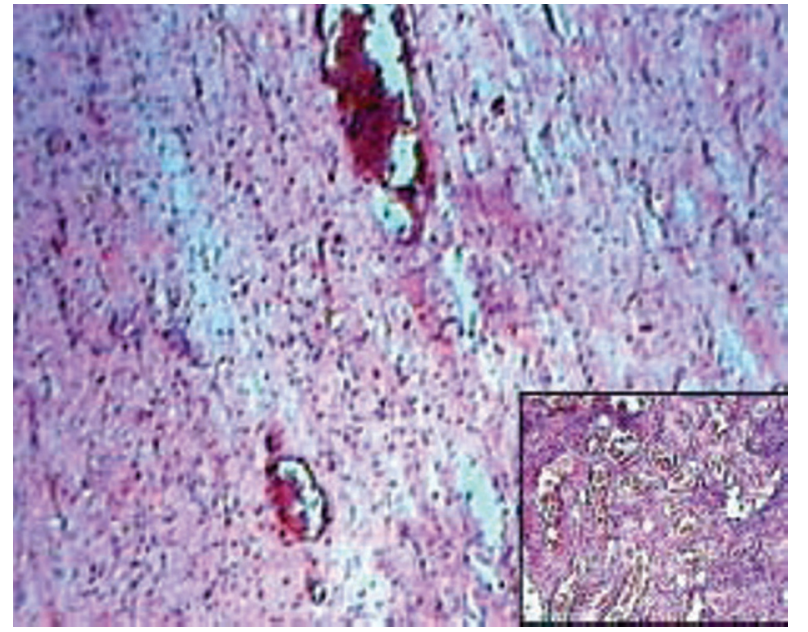

Fig. 2: Microphotograph of aggressive angiomyxoma showing myxoid areas with scattered blood vessels and highly vascular areas showing focal hypercellularity (inset) ( $\mathrm{H} \&$ $\mathrm{E}, \mathrm{x} 150)$

histopathology. They do not recur. Cellular angiofibroma/ spindle cell lipomas are small in size and are mixed with fat with mural hyalinization of blood vessels. Microscopic picture of aggressive angiomyxomas show bland stellate and spindled cells in loose myxoid stroma with blood vessels of various calibers, some of which are widely dilated. Extravasation of red blood cells is seen. Necrosis or mitotic figures are usually not seen. Immunohistochemistry shows focal positivity with vimentin, SMA and desmin. Our case had all the typical clinical and pathological features of an aggressive angiomyxoma. The CD 34 positivity is characteristically seen in such vascular lesions. The case is presented for its rarity and the need for a high index of suspicion in soft tissue tumours occurring in this region.

\section{Conflicts of Interest}

None identified

\section{References}

1. Steeper TA, Rosai J. Aggressive angiomyxoma of female pelvis and perineum. Report of nine cases of a distinct type of gynaecologic soft tissue neoplasm. Am J Surg Pathol 1983; 7:463-75.

2. Behrnwala KA, Thomas JM. Aggressive angiomyxoma: A distinct clinical entity. Eur J Surg Onco 2003; 29: 559-63.

3. Dragoumis K, Drevelengas A, Chatzigeorgiou K, et al. Aggressive angiomyxoma of vulva extending into the pelvis:report of two cases. J Obstet Gynaecol Res 2005 ; 31: 301-3.

4. Chan YM, Hon E, Ngai SW, Ng TY, Wong LC. Aggressive angiomyxoma in female: is radical resection the only option? Acta Obstet Gynecol Scand 2000;79:216-20.

5. Shinora N, Nonomura K, Ishikawa S, Seki H, Koyanagi T. Medical management of recurrent aggressive angiomyxoma with gonadotrophine releasing hormone agonist. Int J Urol 2004; 11:432-5. 